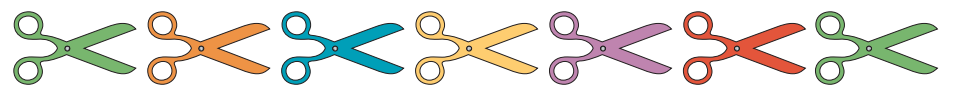

GLYCOPROTEIN DEGRADATION

\section{Time for a trim}

Quality control is essential for cell viability and, in the endoplasmic reticulum (ER), the calnexin/calreticulin chaperone cycle ensures that only correctly folded glycoproteins reach their final destination. Persistently misfolded glycoproteins undergo ERassociated degradation (ERAD); that is, they are retro-translocated to the cytosol and degraded by the proteasome. Previously, EDEM - a transmembrane $\alpha$-mannosidase-I-like protein that lacks mannosidase activity has been shown to accelerate ERAD, and now two Science papers clarify its exact role in this process.

Glycoproteins displaying the $N$ linked glycan $\mathrm{Glc}_{1} \mathrm{Man}_{9} \mathrm{GlcNAc}_{2}$ (where Glc is glucose, Man is mannose and GlcNAc is $N$-acetylglucosamine) bind calnexin or calreticulin, which allows these chaperones to facilitate protein folding, and trimming of the terminal glucose disrupts this interaction. However, if folding is incomplete, the free glycoprotein can be either reglucosylated by a glucosyltransferase to allow further interaction with chaperones or further trimmed by ER $\alpha$-mannosidase I to produce $\mathrm{Man}_{8} \mathrm{GlcNAc}_{2}$, which targets glycoproteins for ERAD.

In the first paper, Nagata and colleagues began by assessing EDEM's interactions. They showed that EDEM interacts with the transmembrane chaperone calnexin, but not with the soluble chaperone calreticulin, and that the transmembrane region of calnexin is required for this interaction.

Next, they used an ERAD substrate called NHK (an $\alpha_{1}$-antitrypsin variant) to study the roles of calnexin and EDEM in ERAD. They compared NHK-transfected cells with cells transfected with NHK and EDEM, and found that NHK degradation was accelerated in the latter. When they disrupted the calnexin-NHK interaction, they found that NHK degradation was only mildly inhibited in NHK-expressing cells, but that the accelerated degradation was strongly suppressed in cells expressing NHK and EDEM. Furthermore, they showed that, in both types of transfected cell, calnexin overexpression suppressed NHK degradation. So, both substrate binding to calnexin and substrate release from calnexin are needed for EDEM-mediated ERAD.

Finally, Nagata and co-workers showed that EDEM overexpression accelerates ERAD by promoting the release of persistently misfolded glycoproteins from calnexin. EDEM therefore seems "...to function in the ERAD pathway by accepting substrates from calnexin".

In the second paper, Molinari et al. showed that EDEM overexpression accelerated the degradation of a membrane-bound and soluble form of an ERAD substrate, without affecting the same substrate lacking $\mathrm{N}$-glycans. They also showed that ERAD-substrate degradation was slower when intracellular EDEM levels were reduced.

When Molinari et al. compared wild-type and EDEM-overexpressing cells, they found that glucose persisted for shorter times on the $\mathrm{N}$-glycans of ERAD substrates in the latter case, which would be expected to affect ERAD-substrate interactions with calnexin and EDEM. Indeed, they showed that, although initial calnexin-ERAD substrate interactions were the same, ERAD substrates dissociated from calnexin faster, and were degraded quicker, in EDEM-overexpressing cells.

Molinari et al. further showed that varying the levels of EDEM did not affect the maturation of correctly folding glycoproteins. EDEM therefore specifically regulates the release of terminally misfolded glycoproteins from the calnexin cycle.

The work of Nagata and colleagues and Molinari et al. has therefore shed light on the role of EDEM in ER quality control. EDEM functions in the ERAD pathway by accepting terminally misfolded glycoproteins from calnexin and, as EDEM levels are upregulated when protein folding is perturbed, it might promote cell recovery at times of ER stress.

Rachel Smallridge

\section{(2) References and links} ORIGINAL RESEARCH PAPERS Oda, $Y$. et al. EDEM as an acceptor of terminally misfolded glycoproteins released from colnexin Science 299 1394-1397 (2003) |Mori, M. et al. Role of EDEM in the release of misfolded glycoproteins from the calnexin cycle. Science 299, 1397-1400 (2003) glycoproteins released from calnexin. Science 299

\section{IN BRIEF}

\section{ADHESION}

TES is a novel focal adhesion protein with a role in cell spreading.

Coutts, A. S. et al. J. Cell Sci. 116, 897-906 (2003)

TES was previously identified as a candidate tumour-suppressor gene. As reported here, Coutts et al. carried out yeast two-hybrid analysis and found that TES interacts with several focal adhesion and/or cytoskeletal proteins. Further studies showed TES to localize to regions of cell-substrate and cell-cell contact. Focaladhesion-associated proteins might mediate cell adhesion, migration or cell signalling, and TES, when overexpressed, was found to confer on fibroblasts an increased ability to spread.

\section{DEVELOPMENT}

Retraction of the Drosophila germ band requires cell-matrix interaction.

Schöck, F. \& Perrimon, N. Genes Dev. 17, 597-602 (2003)

The absence of integrins in Drosophila affects germ-band retraction, which involves large-scale epithelial movements. Here, $\alpha 1,2$ laminin and $\alpha$ PS3 3 PS integrin were shown to be needed for a small group of cells of the amnioserosa (a squamous epithelium) to spread over the end of the germ band. In the absence of $\beta$ PS integrin, lamellipodia formation was inhibited and no cell-matrix adhesion between the amnioserosa and the tail end of the germ band occurred.

\section{SIGNALLING}

Redox-dependent downregulation of Rho by Rac.

Nimnual, A. S., Taylor, L. \& Bar-Sagi, D. Nature Cell Biol. 5, 236-241 (2003)

Balancing the opposing effects of Rac and Rho on the cytoskeleton is crucial for determining cell morphology and migratory behaviour. Here, the authors found that Rac, through its unique insert region, generates reactive oxygen species (ROS). A resultant decrease in Rho activity occurs through ROSmediated inhibition of the low-molecular-weight protein tyrosine phosphatase (LMW-PTP) and subsequent activation, by increased tyrosine phosphorylation, of p190Rho-GAP, enabling Rac to induce membrane ruffling and integrin-mediated spreading.

\section{CHROMOSOME BIOLOGY}

Global chromosome positions are transmitted through mitosis in mammalian cells.

Gerlich, D. et al. Cell 2003 March 4 (DOI: 10.1016/S0092867403001892)

How and when are chromosomes positioned non-randomly in mammalian cells? Gerlich et al. used non-invasive labelling and $4 \mathrm{D}$ imaging to show that no global chromosome rearrangements occur in interphase, G1, S or G2 of the cell cycle, but that global positioning occurs during mitosis and is transmitted from one cell generation to the next. They propose that this occurs by chromosome-specific timing of sister-chromatid separation. 